\title{
Broadband electronically tunable reflection-based phase shifter for active-steering microwave reflectarray systems in Ku-band
}

\author{
Pablo Padilla , Juan F. Valenzuela-Valdés , Jose Luis Padilla , \\ Jose Manuel Fernández-González and Manuel Sierra-Castañer
}

\begin{abstract}
This document provides the design of an electronically reconfigurable microwave phase shifter for reflectarray systems. The phase shifter is based on a hybrid coupler with reflective circuits in three ports. Each reflective circuit introduces a phase variation that can be modified due to the variable capacity value of a varactor inserted in it. The phase shifting process includes three different stages of phase shifting for the incoming signal in its way through the phase shifter: the signal is conducted through the device towards the reflective circuits in four different ways. The input port of the device is also the output one, providing the desired reflective phase shifting effect. This device is of great interest in reflectarray applications in order to provide more than $360^{\circ}$ of controllable phase shifting at each array element. The document includes the complete design of the phase shifter along with its design parameters and circuital behaviour.
\end{abstract}

\section{Introduction}

Reflectarray antennas have become a suitable alternative in communication systems whose requirements impose hard constraints in terms of radiation pattern, directivity and gain, such as satellite communication systems.[1] Traditionally, those systems have included reflector-based antennas in order to achieve the particular features and radiation pattern distributions. The reflectarray concept means the substitution of the conventional conformed reflector. In the case of a reflectarray, the reflective surface that transforms in a plane wave, the quasi-spherical wave provided by the feeder is replaced by a planar array of radiating elements working together with an adequate phase distribution at each array cell.[2] These devices must satisfy demanding requirements related not only to operating issues: radiation pattern, directivity, gain, side lobe level, polarization purity and co-polar/cross-polar ratio, 
but also to mechanical and physical issues: size, compactness, profile, thermal operating range. In the particular case of the steering direction and pattern aspect ratio (side lobe levels, main beam width, etc.), they are controlled by tuning the transmission phase and amplitude of the radiated signal of each array radiating element. The phase shifting strategy, which may vary depending on the design and the final antenna specifications, is typically provided by either a reflective delay line circuit connected to the radiating element or at the radiating element itself by varying the element size and shape.[3]

There is vast experience in the design and manufacturing of passive reflectarrays, whose radiating patterns are initially fixed, not being variable afterwards.[4,5] However, the case of active devices is an ongoing issue.[6,7] This paper presents an electronically tunable phase shifter for reflectarray devices, based on reflective circuits. The tuning effect is introduced by the use of varactors.

This document is organized as follows: Section 2 introduces the phase shifter working principle. Section 3 presents the phase shifter design and Section 4 provides the design performance. Finally, conclusions are drawn in Section 5.

\section{Phase shifter working principle and circuital behaviour}

The phase shifting strategy for reflectarray structures implies the use of reflective designs with the same port acting as input and output port. When considering reflective LC circuits, one of the main drawbacks is the impossibility of obtaining a complete $360^{\circ}$ phase variation and also the lack of linearity in the relation between the polarization voltage and the phase shift.[8] The approach provided in this work implies the use of a hybrid 3- $\mathrm{dB} / 90^{\circ}$ coupler in order to sequentially add the phase shift to the signal in its progression through the shifter, using reflective circuits in the different coupler ports. Figure 1 depicts the shifter functioning. Considering that port 1 is the input port, ports 3 and 4 are the coupled output ports with a difference of $90^{\circ}$ and port 2 is the isolated port, the proposed operating scheme is as follows: the input signal in port 1 propagates towards ports 3 and 4 with a phase difference of $90^{\circ}$ (way 1). In those ports, the signals are delayed according to the reflective circuits (first shifting stage) and reflected towards port 2 (way 2), where the signals coming from ports 3 and 4 are combined in phase (the $90^{\circ}$ difference is reverted) and the resulting signal is again

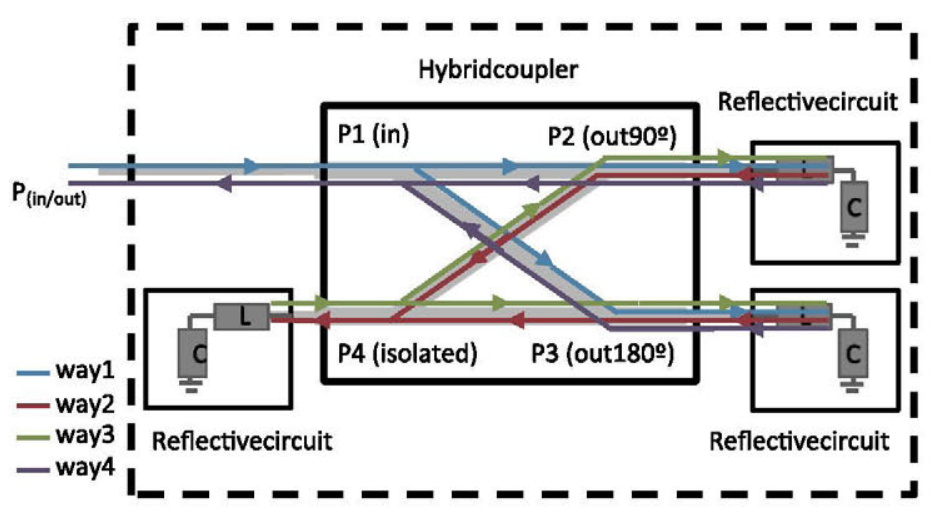

Figure 1. Schematic working principle of the reflective phase shifter. 


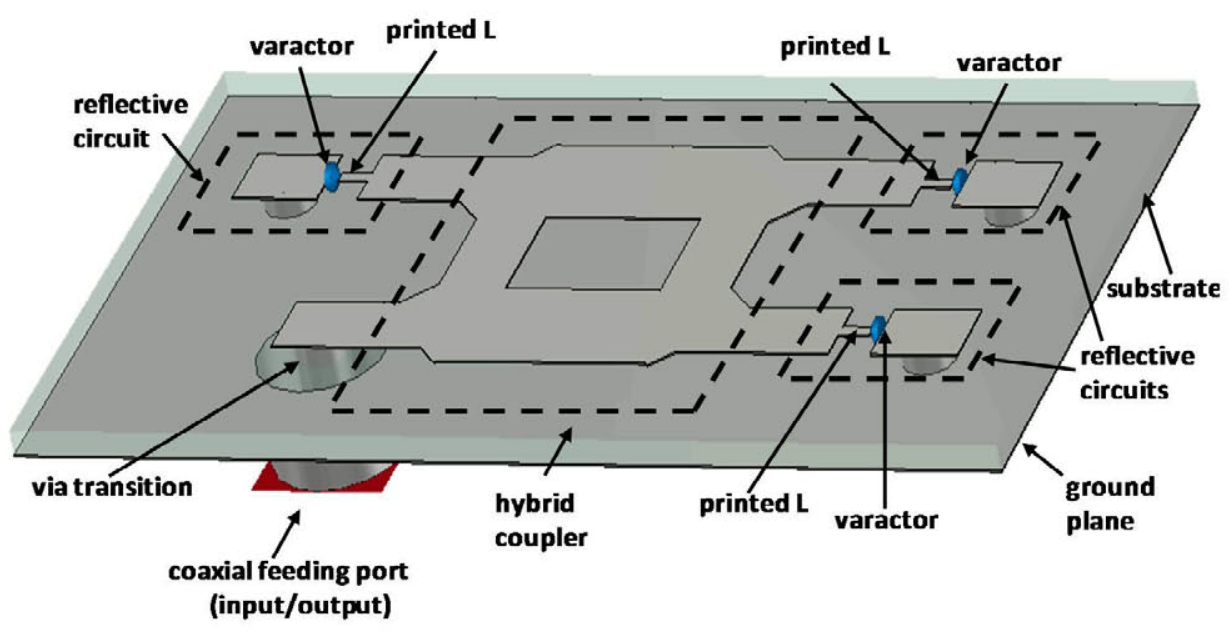

Figure 2. Design model of the reflective phase shifter.

delayed due to an additional reflective circuit (second shifting stage). Then, the signal is redirected once more towards port 3 and 4 (way 3), where once more a phase variation is added (third shifting stage), and finally sent towards port 1 as the output of the device (way 4).

\section{Device design}

The particular topology in which the shifter is intended to be placed needs to be assessed. The reflectarray structure implies the use of a radiating layer and a shifting layer, both separated by a ground plane. The connection between these two layers may be performed either with direct via connection or slot coupling. The first one provides more bandwidth and reliability. Thus, the proposed design has an input/output port based on a metallic via through a hole in the ground plane.

Figure 2 provides the design model of the proposed design for the reflective phase shifter. After an optimization process, the final design parameters and dimensions are obtained, as provided in Table 1. The final electromagnetic characterization has been carried out with CST MWS.

The basic element of the tunable phase shifter, the hybrid coupler, is printed over the upper side of a Neltec substrate (NY217, $\varepsilon r=2.17, h=0.508 \mathrm{~mm}, \tan \delta=0.0002$ ), whereas the lower side is the copper ground plane. Three of its ports are ended with reflective RF circuits. These ones are formed by an equivalent LC circuit with a printed inductance and a varactor with variable capacity value, joined to the ground plane through a metallic via.

\section{Design evaluation}

The phase shifter provides the working outcomes provided in Figures 3 and 4 in terms of: phase shifting of the reflective circuits (Figure 3), phase shifting of the complete device (Figure 4(a)) and complete shifting circuit losses (Figure 4(b)). Notice that a phase variation 
Table 1. Design parameters and dimensions.

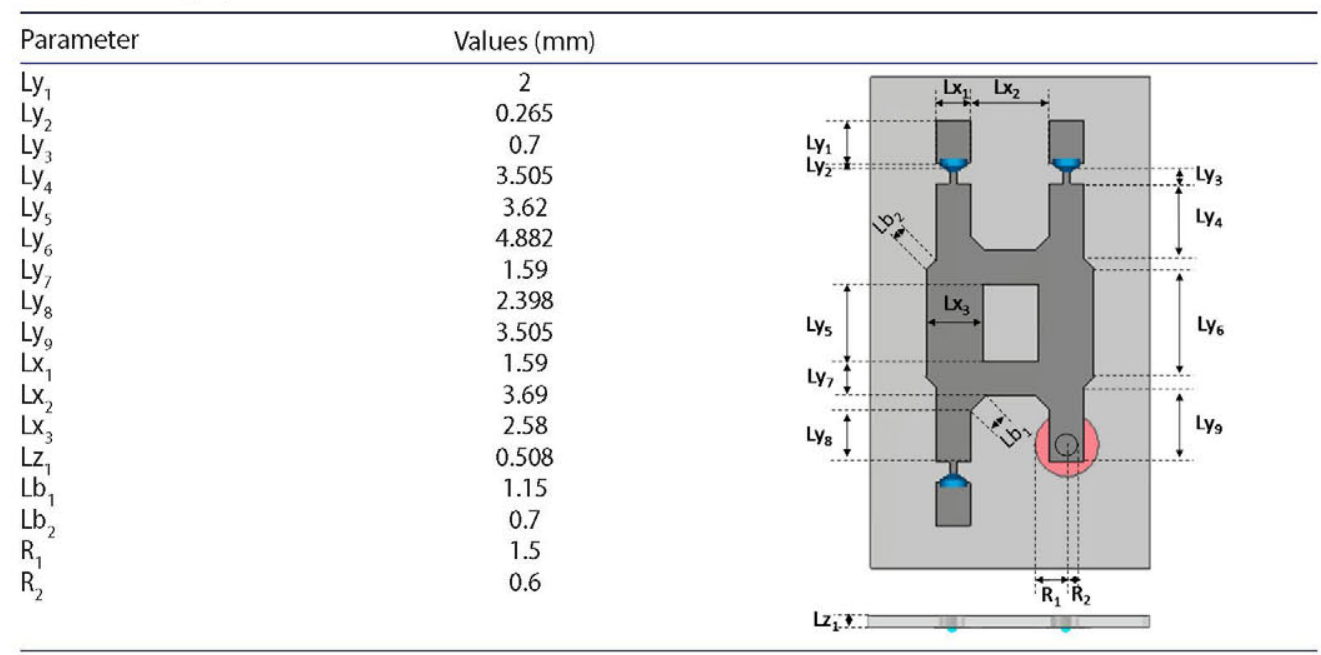

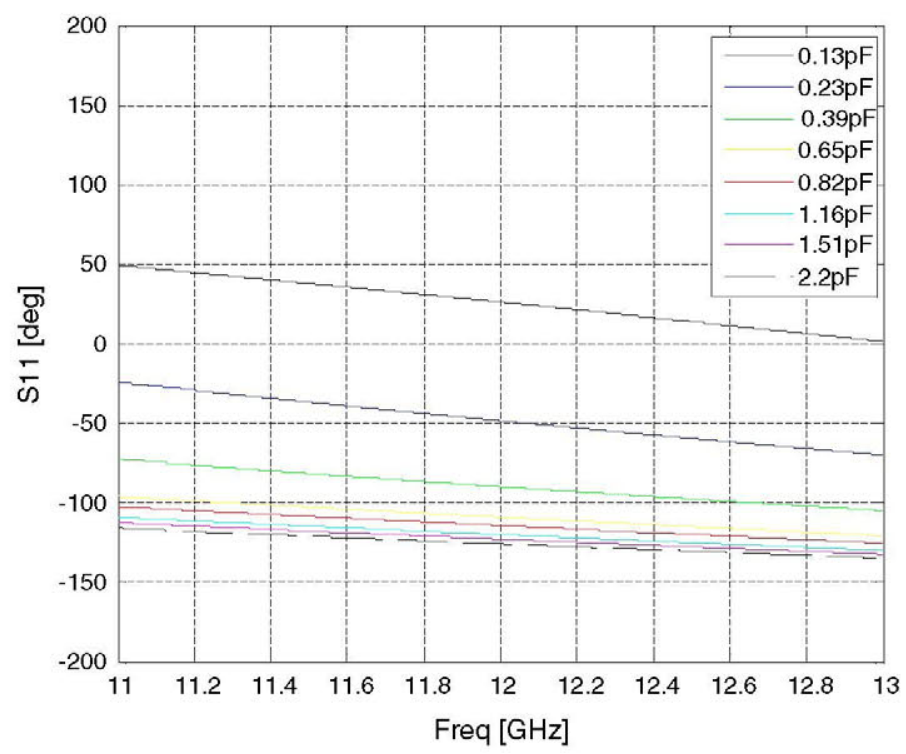

Figure 3. $\arg \left(\mathrm{S}_{11}\right)$ of the reflective LC circuit.

of around $480^{\circ}$ is obtained, much more than the required $360^{\circ}$, to guarantee phase wrapping at each element of the reflectarray structure.

As it can be noticed, the phase shift provided by the reflective circuits $\left(\sim 180^{\circ}\right.$ of phase variation) constitutes sequentially the complete phase shift of around $480^{\circ}$ of the final device. It can be observed that the phase variation is not linear with the capacity value (Figures 3 and $4(\mathrm{a})$ ). However, the additional non-linear response regarding polarization voltage vs. 

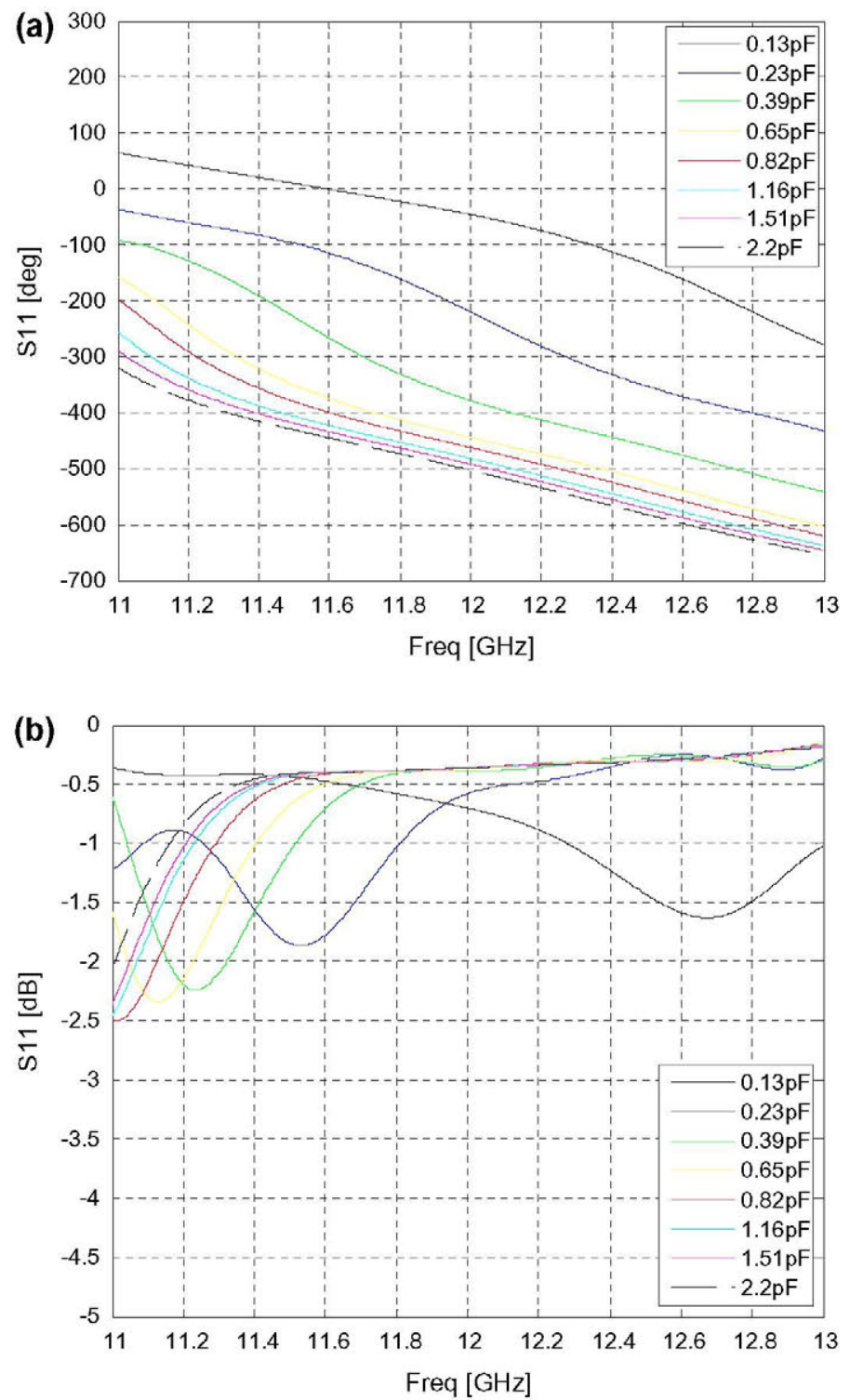

Figure 4. Performance results of the microwave reflective phase shifter, $(a) \arg \left(S_{11}\right)$, (b) $\left|S_{11}\right|$.

capacity value makes that, when both behaviours are combined, the final phase shift vs. voltage relation yields linearity in almost all the capacity range of the varactor. This linear range provides more than $360^{\circ}$ in phase shifting. Figure 5 shows this fact. Regarding the circuit losses, they are below $2 \mathrm{~dB}$ in all the working range, considering the worst case (Figure 4(b)).

This phase shifter design provides compactness and a reduction in the number of lumped elements, compared to other designs available in the literature, and fits the size requirements of the reflectarray systems. 


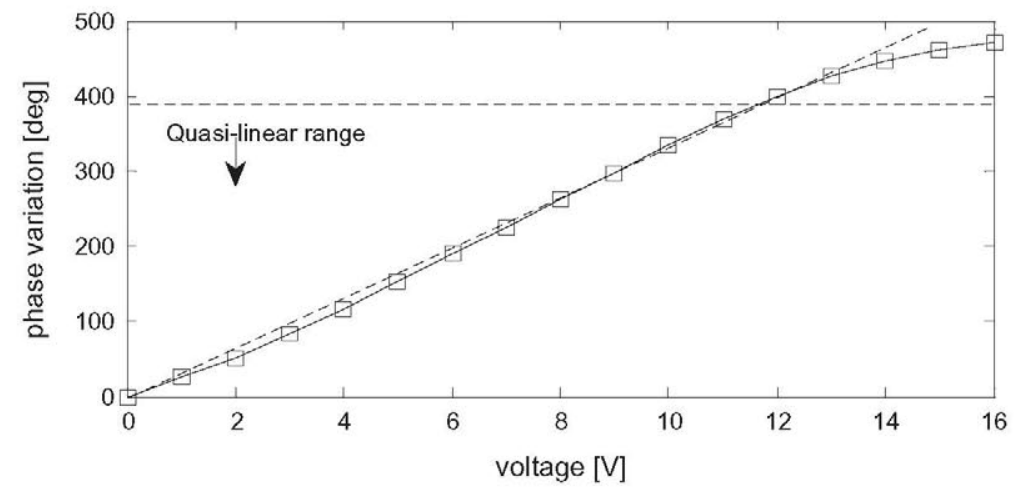

Figure 5. Phase shifter circuital behaviour: phase variation vs. voltage.

\section{Conclusion}

This document presents the design of a phase shifter for active reflectarray systems at microwave frequencies. The phase shifter is based on a $3-\mathrm{db} / 90^{\circ}$ coupler with reflective circuits in three of its four ports. Each reflective circuit is composed of a printed inductance and a voltage-controlled varactor. The phase shifting process includes three different stages of phase shifting for the signal in its way through the phase shifter. The complete device yields a phase variation higher than $480^{\circ}$ and more than $360^{\circ}$ in the linear zone. The complete proposed design, along with its performance in terms of matching, losses and phase shifting, is provided in this document. The device design reported in this work reduces the complexity in the integration of active shifting at each unitary cell of the reflectarray structure.

\section{Disclosure statement}

No potential conflict of interest was reported by the authors.

\section{Funding}

This work has been supported by the [TEC2014-55735-C3-1-R] of the Spanish National Program of Research, Development and Innovation. The electromagnetic design of the unitary cell has been developed with CST Studio Suite under licensed agreement between Computer Simulation Technology (CST) and Technical University of Madrid.

\section{References}

[1] Huang J, Encinar JA. Reflectarray antennas. Ed. Wiley; 2008.

[2] Nayeri P, Yang F, Elsherbeni AZ. Beam-scanning reflectarray antennas: a technical overview and state of the art. IEEE Antennas Propag. Mag. 2015;57:32-47.

[3] Tienda C, Encinar JA, Arrebola M, et al. Design, manufacturing and test of a dual-reflectarray antenna with improved bandwidth and reduced cross-polarization. IEEE Trans. Antennas Propag. 2013;61:1180-1190.

[4] Carrasco E, Barba M, Encinar JA. Aperture-coupled reflectarray element with wide range of phase delay. Electron. Lett. 2006;42:667-668. 
[5] Padilla JL, Padilla P, Valenzuela-Valdés JF, et al. High-frequency radiating element and modified $3 \mathrm{~dB} / 90^{\circ}$ electronic shifting circuit with circular polarization for broadband reflectarray device cells. Electron. Lett. 2014;50:1042-1043.

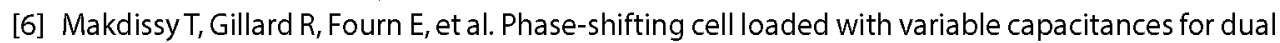
linearly polarised reflectarrays. Electron. Lett. 2012;48:1319-1320.

[7] Venneri $F$, Costanzo S, Di Massa G. Design and validation of a reconfigurable single varactor-tuned reflectarray. IEEE Trans. Antennas Propag. 2013;61:635-645.

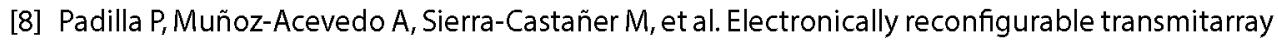
at Ku band for microwave applications. IEEE Trans. Antennas Propag. 2010;58:2571-2579. 